\title{
Oxidation of sulfides to chiral sulfoxides using Schiff base-vanadium (IV) complexes
}

\author{
Ángeles Gama, Lucía Z. Flores-López, Gerardo Aguirre, Miguel Parra-Hake, \\ Lars H. Hellberg, and Ratnasamy Somanathan*
}

Centro de Graduados e Investigación, Instituto Tecnológico de Tijuana, Apdo. Postal 1166, 22000, Tijuana, B.C. México

E-mail:somanathan@tectijuana.mx

(received 03 Jun 03; accepted 05 Sep 03; published on the web 09 Sep 03)

\begin{abstract}
A library of Schiff base ligands was synthesized from salicylaldehyde by reaction with various $\beta$-amino alcohols. These ligands were used with vanadium (IV) to screen for the enantioselective oxidation of sulfides to chiral sulfoxides.
\end{abstract}

Keywords: Sulfide, chiral sulfoxide, Schiff base, vanadium (IV) catalyst

\section{Introduction}

An increasing number of chiral sulfoxides are becoming useful chiral auxiliaries in asymmetric synthesis. $^{1-6}$ Satoh and coworkers have reported the synthesis of chiral allenes by first coupling alkenyl aryl sulfoxides with aldehydes followed by alkyl anion induced elimination of the sulfur. ${ }^{7}$ Toru has reported the enantioselective addition of Grignard reagents to 1-(arylsulfinyl)-2naphthaldehyde, where a chiral sulfoxide conformer controls stereoselectivity of the addition. ${ }^{8}$ Optically active $\beta$-(trimethylsilyl)ethyl sulfoxides supported on Merrifield resin undergo enantioselective Michael addition to $\alpha, \beta$-unsturated esters, followed by removal of the sulfoxide group via thermal elimination. ${ }^{9}$

Recently a number of reports have appeared describing the use of chiral alkene and diene sulfoxides in synthetically-useful Diels-Alder reactions, where the chiral sulfoxide plays a major role in inducing chirality in the final product. ${ }^{10-13}$ Yuste and Ellman have independently described the use of sulfoxides as chiral auxilaries in the asymmetric synthesis of $\beta$-amino alcohols which, in turn are synthetically-useful chiral building blocks. ${ }^{14 a, b, ~ c, ~ 15 a, ~ b ~}$ Toru has reported the elegant use of a chiral sulfoxide to synthesize an insecticidal chiral chrysanthamate. ${ }^{16 a}$ More recently, Colobert $^{16 \mathrm{~b}}$ and Bravo ${ }^{16 \mathrm{c}}$ have demonstrated the use of chiral sufoxides in the synthesis of myoinositol, pyrrolidine and tetrahydroisoquinoline alkaloids, respectively. These examples clearly demonstrate the versatility of chiral sulfoxides as chiral auxilaries in asymmetric synthesis. 
A number of sulfoxides are also finding application in the pharmaceutical industry. The chiral sulfoxide quinolone $\mathbf{1}$ is known to inhibit platelet adhesion by interfering with the release of 12(S)-hydroxyeicosatetraenoic acid from platelets. ${ }^{17 a, b,} 18$ Pyrazolotriazine 2 is a new drug developed to treat hyperuricemia and isochemic reperfusion injury. The drug inhibits the biosynthesis of uric acid by blocking xanthine oxidase. ${ }^{19}$ Unge and co-workers have reported the asymmetric synthesis of esomeprazole, a drug containing a chiral sulfoxide group known to inhibit gastric acid secretion. ${ }^{20 a}$ Padmanahan and co-workers from Cambridge Neuro Science have reported the asymmetric synthesis of a sulfoxide containing a guanidine portion that is an active $N$-methyl-D-aspartate ion-channel blocker. ${ }^{20 \mathrm{~b}}$ These few examples clearly illustrate the growing importance of chiral sulfoxides in the pharmaceutical industry.

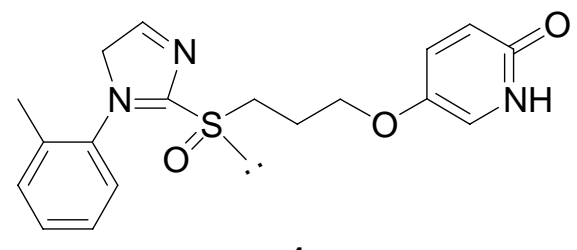

1

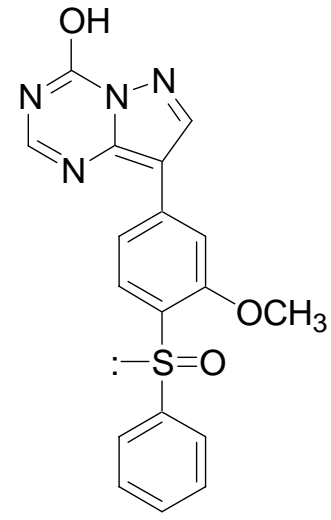

2

Since enantiomerically pure sulfoxides can play an important role as chiral auxilaries in organic synthesis, it is surprising that very few examples exist in which this ligand participates in homogeneous catalysis. Khiar used a Fe(III) complex of $\mathrm{C}_{2}$-symmetric bis-sulfoxide as a catalyst in the asymmetric Diels-Alder reaction. ${ }^{21}$ Shibasaki and Williams have independently used Pdsulfoxide complexes in asymmetric allylic substitution. ${ }^{22,23}$ Bolm and Carreño have also attempted the use of chiral sulfoxides to catalyse the enantioselective addition of diethyl zinc to aromatic aldehydes; the products were obtained in moderate ee's. ${ }^{24,25}$

These results have prompted researchers over the past two decades to develop new methods leading to asymmetric oxidation of a sulfide to a chiral sulfoxide (Equation 1).

$$
R^{-\ddot{S} \cdot R^{\prime}} \longrightarrow R^{\prime \prime \prime} S^{\prime \prime} R^{\prime}
$$


Numerous methodologies have been reported for the transformation of a prochiral sulfide to a chiral sulfoxide. Most of them involve use of a chiral ligand with a transition metal, such as titanium, vanadium or manganese, in the presence of hydrogen peroxide or an hydrogen peroxide adduct as the oxygen source. The chiral ligands that have been successfully used include: bidentate diethyl tartrate $\mathbf{3},{ }^{26}$ diol $\mathbf{4},{ }^{27}$ BINOL $\mathbf{5},{ }^{28,}{ }^{29}$ tridentate Schiff base ligands $\mathbf{6}^{30-32}$ and tetradentate Salen type ligands 7 . $^{33-36}$<smiles>CCOC(=O)C(O)C(O)C(=O)OCC</smiles>

3<smiles>OC(Br)C(O)Br</smiles>

4<smiles>Oc1ccc2ccccc2c1-c1c(O)ccc2ccccc12</smiles>

5

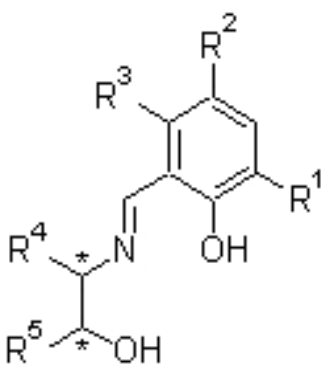

6<smiles>[R]c1cccc(C=N[C@@H]([R])C([R])/N=C/c2cccc([R])c2O)c1O</smiles>

7

\section{Results and Discussion}

As part of a wider study of asymmetric transformations, we proposed the preparation of a large library of chiral Schiff base ligands of the -O---N---O- type 6. Along with a transition metal ion, Ti(IV), V(IV), Cu(II) or Zn(II)), it would permit screening of the Schiff base ligands in various asymmetric chemical transformations. Recent application of this strategy in our laboratories to the addition of trimethylsilyl cyanide to benzaldehyde in the presence of Ti (IV) ion resulted in trimethylsilyl cyanohydrins in 40-85\% enantioselectivity (Equation 2). ${ }^{37,38}$

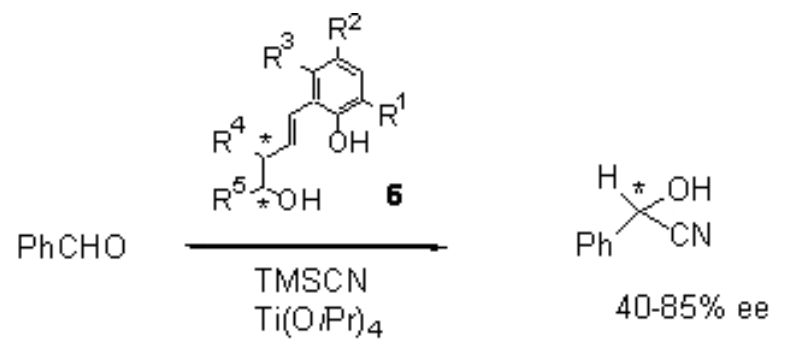


Vanadium (IV)-Schiff base complexes have been successfully used by Bolm, ${ }^{31}$ Ellman ${ }^{15}$ and Skarzewski ${ }^{32}$ to oxidize different sulfide substrates to chiral sulfoxides. Based on these reports we have created a library of Schiff base ligands with subtle variations in the size of the substituents on the ligand. The library of ligands was derived from salicylaldehydes $\mathbf{8}$ and chiral $\beta$-amino alcohols 9 as shown in Equation 3.

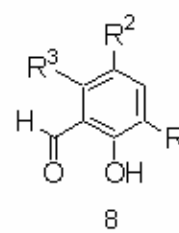

$$
\begin{aligned}
& \mathrm{R}^{1}=\mathrm{R}^{2}=\mathrm{C}\left(\mathrm{CH}_{3}\right)_{3}, \mathrm{R}^{3}=\mathrm{H} \\
& \mathrm{R}^{1}=\mathrm{C}\left(\mathrm{CH}_{3}\right)_{3}, \mathrm{R}^{2}=\mathrm{R}^{3}=\mathrm{H} \\
& \mathrm{R}^{1}=\mathrm{R}^{2}=\mathrm{CH}_{3}, \mathrm{R}^{3}=\mathrm{H} \\
& \mathrm{R}^{1}=\mathrm{Adamantyl}, \mathrm{R}^{2}=\mathrm{CH}_{3}, \mathrm{R}^{3}=\mathrm{H} \\
& \mathrm{R}^{1}=\mathrm{R}^{2}=\mathrm{C}\left(\mathrm{CH}_{3}\right)_{2} \mathrm{Ph}_{1} \mathrm{R}^{3}=\mathrm{H} \\
& \mathrm{R}^{1}=\mathrm{R}^{2}=\mathrm{C}\left(\mathrm{CH}_{3}\right)_{2} \mathrm{CH}_{2} \mathrm{CH}_{3}, \mathrm{R}^{3}=\mathrm{H} \\
& \mathrm{R}^{1}=\mathrm{OCH}_{3}, \mathrm{R}^{2}=\mathrm{R}^{3}=\mathrm{H} \\
& \mathrm{R}^{1}=\mathrm{C}\left(\mathrm{CH}_{3}\right)_{3}, \mathrm{R}^{2}=\mathrm{NO}_{2}, \mathrm{R}^{3}=\mathrm{H} \\
& \mathrm{R}^{1}=\mathrm{H}, \mathrm{R}^{2}=\mathrm{NO}_{2}, \mathrm{R}^{3}=\mathrm{H} \\
& \mathrm{R}^{1}=\mathrm{C}\left(\mathrm{CH}_{3}\right)_{3}, \mathrm{R}^{2}=\mathrm{Br}, \mathrm{R}^{3}=\mathrm{H} \\
& \mathrm{R}^{1}=\mathrm{R}^{2}=\mathrm{Br}, \mathrm{R}^{3}=\mathrm{H} \\
& \mathrm{R}^{1}=\mathrm{H}, \mathrm{R}^{2}=\mathrm{Br}, \mathrm{R}^{3}=\mathrm{H} \\
& \mathrm{R}^{1}=\mathrm{H}, \mathrm{R}^{2}=\mathrm{R}^{3}=\mathrm{Ph}
\end{aligned}
$$

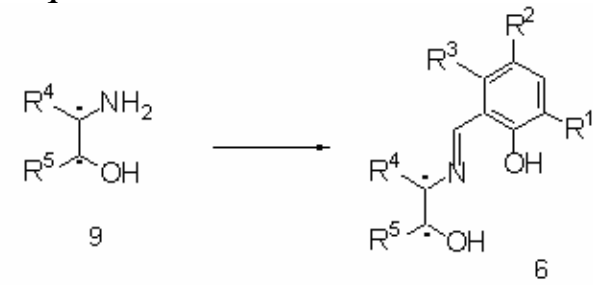

$\mathrm{R}^{4}=\mathrm{R}^{5}=\mathrm{CH}_{2} \mathrm{C}_{6} \mathrm{H}_{4}$ $\mathrm{R}^{4}=\mathrm{C}\left(\mathrm{CH}_{3}\right)_{3}, \mathrm{R}^{5}=\mathrm{H}$ $R^{4}=P h, R^{5}=H$ $\mathrm{R}^{4}=\mathrm{R}^{5}=\mathrm{Ph}$ $\mathrm{R}^{4}=\mathrm{CH}\left(\mathrm{CH}_{3}\right)_{2}, \mathrm{R}^{5}=\mathrm{H}$

$\mathrm{R}^{4}=\mathrm{CH}_{2} \mathrm{CH}\left(\mathrm{CH}_{3}\right)_{2}, \mathrm{R}^{5}=\mathrm{H}$ $\mathrm{R}^{4}=\mathrm{CH}_{3}, \mathrm{R}^{5}=\mathrm{H}$ $\mathrm{R}^{4}=\mathrm{H}_{1} \mathrm{R}^{5}=\mathrm{CH}_{3}$ $\mathrm{R}^{4}=\mathrm{CH}_{2} \mathrm{CH}_{3}, \mathrm{R}^{5}=\mathrm{H}$ $\mathrm{R}^{4}=\mathrm{CHCH}_{3} \mathrm{CH}_{2} \mathrm{CH}_{3}, \mathrm{R}^{5}=\mathrm{H}$

The results of our screening are shown in Table 1. From our previous work with these ligands in the trimethylsilylcyanation of benzaldehyde catalyzed by Ti(IV)-Schiff base complexes, we discovered it was necessary to have a bulky substituent ortho to the phenol $\left(\mathrm{R}_{1}\right)$. ${ }^{37}$ A similar trend was also observed in the sulfide oxidation; when $\mathrm{R}_{1}=\mathrm{H}, \mathrm{OCH}_{3}$ or $\mathrm{R}_{2}, \mathrm{R}_{3}=$ naphthyl, the observed ee's were low. Hence, we designed a number of Schiff bases with a bulky substituent at $R_{1}$, and then varied the size of substituents on $R_{2}, R_{4}$ and $R_{5}$. Initially, we incorporated a conformationally-rigid five membered ring at $\mathrm{R}_{4}$ and $\mathrm{R}_{5}$, derived from cis-1amino-2-indanol. Our assumption here was that the bulky indanol ring would increase the energy difference between two diastereomeric transition structure orientations, thereby enhancing the resulting enantioselectivity. When $\mathrm{R}_{1}=$ tert-butyl or adamantyl, and $\mathrm{R}_{4}, \mathrm{R}_{5}=$ cis-1-amino-2indanyl, reasonably good enantioselectivities were observed (ligand $\mathbf{1 0}$ ). However, when $\mathrm{R}_{1}$ was replaced with 3,3-dimethyl propyl or 1,1-dimethylbenzyl, enantioselectivity was considerably lower (ligands 15 and 16). This lowering of enantioselectivity probably came from steric overcrowding around the metal, thereby inhibiting the sulfide-metal coordination. Interestingly, when the rigid five-membered ring was replaced with a conformationally more flexible $\beta$-amino alcohol fragment $\left(\mathrm{R}_{4}\right)$, enantioselectivity was considerably improved, (ligands 42 , 43 and 44). However, when both $R_{4}$ and $R_{5}$ were substituted, the enantioselectivity once again decreased (ligands 28-32). Our results are in accordance with the recent report from Bergman and Ellman, ${ }^{14 a}$ who have 
Table 1. Enantioselective catalytic oxidation of sulfide to sulfoxides promoted by chiral Schiff base-vanadium(IV) complexes derived from $\beta$-aminoalcohols

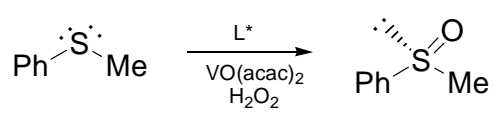

\begin{tabular}{|c|c|c|c|c|c|c|c|}
\hline $\begin{array}{c}\text { Schiff } \\
\text { Base }\end{array}$ & $\mathbf{R}^{1}$ & $\mathbf{R}^{2}$ & $\mathbf{R}^{3}$ & $\mathbf{R}^{4}$ & $\mathbf{R}^{5}$ & $\begin{array}{c}\% \\
\text { Yield } \\
\end{array}$ & $\begin{array}{c}\text { \% e.e. } \\
\text { (Configu } \\
\text { ration) }\end{array}$ \\
\hline 10 & Adamantyl & $\mathrm{CH}_{3}$ & $\mathrm{H}$ & \multicolumn{2}{|l|}{$\mathrm{CH}_{2} \mathrm{C}_{6} \mathrm{H}_{4}$} & 54 & $50(R)$ \\
\hline 11 & $\mathrm{OCH}_{3}$ & $\mathrm{H}$ & $\mathrm{H}$ & \multicolumn{2}{|l|}{$\mathrm{CH}_{2} \mathrm{C}_{6} \mathrm{H}_{4}$} & 56 & $38(R)$ \\
\hline 12 & $\mathrm{H}$ & $\mathrm{NO}_{2}$ & $\mathrm{H}$ & \multicolumn{2}{|l|}{$\mathrm{CH}_{2} \mathrm{C}_{6} \mathrm{H}_{4}$} & 88 & $24(S)$ \\
\hline 13 & $\mathrm{CH}_{3}$ & $\mathrm{CH}_{3}$ & $\mathrm{H}$ & \multicolumn{2}{|l|}{$\mathrm{CH}_{2} \mathrm{C}_{6} \mathrm{H}_{4}$} & 61 & $38(S)$ \\
\hline 14 & $\mathrm{C}\left(\mathrm{CH}_{3}\right)_{3}$ & $\mathrm{NO}_{2}$ & $\mathrm{H}$ & \multicolumn{2}{|l|}{$\mathrm{CH}_{2} \mathrm{C}_{6} \mathrm{H}_{4}$} & 50 & $45(S)$ \\
\hline 15 & $\mathrm{C}\left(\mathrm{CH}_{3}\right)_{2} \mathrm{CH}_{2} \mathrm{CH}_{3}$ & $\mathrm{C}\left(\mathrm{CH}_{3}\right)_{2} \mathrm{CH}_{2} \mathrm{CH}_{3}$ & $\mathrm{H}$ & \multicolumn{2}{|l|}{$\mathrm{CH}_{2} \mathrm{C}_{6} \mathrm{H}_{4}$} & 90 & $22(S)$ \\
\hline 16 & $\mathrm{C}\left(\mathrm{CH}_{3}\right)_{2} \mathrm{Ph}$ & $\mathrm{C}\left(\mathrm{CH}_{3}\right)_{2} \mathrm{Ph}$ & $\mathrm{H}$ & \multicolumn{2}{|l|}{$\mathrm{CH}_{2} \mathrm{C}_{6} \mathrm{H}_{4}$} & 51 & $18(S)$ \\
\hline 17 & $\mathrm{Br}$ & $\mathrm{Br}$ & $\mathrm{H}$ & \multicolumn{2}{|l|}{$\mathrm{CH}_{2} \mathrm{C}_{6} \mathrm{H}_{4}$} & 88 & $61(R)$ \\
\hline 18 & $\mathrm{C}\left(\mathrm{CH}_{3}\right)_{3}$ & $\mathrm{Br}$ & $\mathrm{H}$ & \multicolumn{2}{|l|}{$\mathrm{CH}_{2} \mathrm{C}_{6} \mathrm{H}_{4}$} & 83 & $51(R)$ \\
\hline 19 & $\mathrm{C}\left(\mathrm{CH}_{3}\right)_{3}$ & $\mathrm{H}$ & $\mathrm{H}$ & \multicolumn{2}{|l|}{$\mathrm{CH}_{2} \mathrm{C}_{6} \mathrm{H}_{4}$} & 64 & $45(R)$ \\
\hline 20 & Adamantyl & $\mathrm{CH}_{3}$ & $\mathrm{H}$ & $\mathrm{Ph}$ & $\mathrm{H}$ & 63 & $42(R)$ \\
\hline 21 & $\mathrm{C}\left(\mathrm{CH}_{3}\right)_{2} \mathrm{CH}_{2} \mathrm{CH}_{3}$ & $\mathrm{C}\left(\mathrm{CH}_{3}\right)_{2} \mathrm{CH}_{2} \mathrm{CH}_{3}$ & $\mathrm{H}$ & $\mathrm{Ph}$ & $\mathrm{H}$ & 62 & $44(R)$ \\
\hline 22 & $\mathrm{C}\left(\mathrm{CH}_{3}\right)_{2} \mathrm{Ph}$ & $\mathrm{C}\left(\mathrm{CH}_{3}\right)_{2} \mathrm{Ph}$ & $\mathrm{H}$ & $\mathrm{Ph}$ & $\mathrm{H}$ & 66 & $28(R)$ \\
\hline 23 & $\mathrm{C}\left(\mathrm{CH}_{3}\right)_{3}$ & $\mathrm{C}\left(\mathrm{CH}_{3}\right)_{3}$ & $\mathrm{H}$ & $\mathrm{Ph}$ & $\mathrm{H}$ & 42 & $35(R)$ \\
\hline 24 & $\mathrm{H}$ & $\mathrm{Br}$ & $\mathrm{H}$ & $\mathrm{Ph}$ & $\mathrm{H}$ & 92 & $16(R)$ \\
\hline 25 & $\mathrm{Br}$ & $\mathrm{Br}$ & $\mathrm{H}$ & $\mathrm{Ph}$ & $\mathrm{H}$ & 48 & $36(R)$ \\
\hline 26 & $\mathrm{C}\left(\mathrm{CH}_{3}\right)_{3}$ & $\mathrm{Br}$ & $\mathrm{H}$ & $\mathrm{Ph}$ & $\mathrm{H}$ & 76 & $29(R)$ \\
\hline 27 & $\mathrm{H}$ & \multicolumn{2}{|l|}{$\mathrm{Ph}$} & $\mathrm{Ph}$ & $\mathrm{H}$ & 61 & $9(R)$ \\
\hline 28 & $\mathrm{C}\left(\mathrm{CH}_{3}\right)_{3}$ & $\mathrm{Br}$ & $\mathrm{H}$ & $\mathrm{Ph}$ & $\mathrm{Ph}$ & 73 & $24(R)$ \\
\hline 29 & Adamantyl & $\mathrm{CH}_{3}$ & $\mathrm{H}$ & $\mathrm{Ph}$ & $\mathrm{Ph}$ & 66 & $35(R)$ \\
\hline 30 & $\mathrm{C}\left(\mathrm{CH}_{3}\right)_{2} \mathrm{CH}_{2} \mathrm{CH}_{3}$ & $\mathrm{C}\left(\mathrm{CH}_{3}\right)_{2} \mathrm{CH}_{2} \mathrm{CH}_{3}$ & $\mathrm{H}$ & $\mathrm{Ph}$ & $\mathrm{Ph}$ & 80 & $38(R)$ \\
\hline 31 & $\mathrm{C}\left(\mathrm{CH}_{3}\right)_{2} \mathrm{Ph}$ & $\mathrm{C}\left(\mathrm{CH}_{3}\right)_{2} \mathrm{Ph}$ & $\mathrm{H}$ & $\mathrm{Ph}$ & $\mathrm{Ph}$ & 91 & $15(R)$ \\
\hline 32 & $\mathrm{C}\left(\mathrm{CH}_{3}\right)_{3}$ & $\mathrm{C}\left(\mathrm{CH}_{3}\right)_{3}$ & $\mathrm{H}$ & $\mathrm{Ph}$ & $\mathrm{Ph}$ & 64 & $28(R)$ \\
\hline 33 & Adamantyl & $\mathrm{CH}_{3}$ & $\mathrm{H}$ & $\mathrm{CH}_{2} \mathrm{CH}\left(\mathrm{CH}_{3}\right)_{2}$ & $\mathrm{H}$ & 65 & $41(S)$ \\
\hline 34 & $\mathrm{C}\left(\mathrm{CH}_{3}\right)_{2} \mathrm{CH}_{2} \mathrm{CH}_{3}$ & $\mathrm{C}\left(\mathrm{CH}_{3}\right)_{2} \mathrm{CH}_{2} \mathrm{CH}_{3}$ & $\mathrm{H}$ & $\mathrm{CH}_{2} \mathrm{CH}\left(\mathrm{CH}_{3}\right)_{2}$ & $\mathrm{H}$ & 66 & $24(S)$ \\
\hline 35 & $\mathrm{C}\left(\mathrm{CH}_{3}\right)_{2} \mathrm{Ph}$ & $\mathrm{C}\left(\mathrm{CH}_{3}\right)_{2} \mathrm{Ph}$ & $\mathrm{H}$ & $\mathrm{CH}_{2} \mathrm{CH}\left(\mathrm{CH}_{3}\right)_{2}$ & $\mathrm{H}$ & 77 & $20(S)$ \\
\hline 36 & $\mathrm{C}\left(\mathrm{CH}_{3}\right)_{3}$ & $\mathrm{C}\left(\mathrm{CH}_{3}\right)_{3}$ & $\mathrm{H}$ & $\mathrm{CHCH}_{3} \mathrm{CH}_{2} \mathrm{CH}_{3}$ & $\mathrm{H}$ & 60 & $37(S)$ \\
\hline 37 & Adamantyl & $\mathrm{CH}_{3}$ & $\mathrm{H}$ & $\mathrm{CH}\left(\mathrm{CH}_{3}\right)_{2}$ & $\mathrm{H}$ & 56 & $43(S)$ \\
\hline 38 & $\mathrm{C}\left(\mathrm{CH}_{3}\right)_{3}$ & $\mathrm{C}\left(\mathrm{CH}_{3}\right)_{3}$ & $\mathrm{H}$ & $\mathrm{CH}\left(\mathrm{CH}_{3}\right)_{2}$ & $\mathrm{H}$ & 58 & $37(R)$ \\
\hline 39 & $\mathrm{C}\left(\mathrm{CH}_{3}\right)_{2} \mathrm{CH}_{2} \mathrm{CH}_{3}$ & $\mathrm{C}\left(\mathrm{CH}_{3}\right)_{2} \mathrm{CH}_{2} \mathrm{CH}_{3}$ & $\mathrm{H}$ & $\mathrm{C}\left(\mathrm{CH}_{3}\right)_{3}$ & $\mathrm{H}$ & 41 & $49(S)$ \\
\hline 40 & $\mathrm{C}\left(\mathrm{CH}_{3}\right)_{2} \mathrm{Ph}$ & $\mathrm{C}\left(\mathrm{CH}_{3}\right)_{2} \mathrm{Ph}$ & $\mathrm{H}$ & $\mathrm{C}\left(\mathrm{CH}_{3}\right)_{3}$ & $\mathrm{H}$ & 83 & $50(S)$ \\
\hline 41 & Adamantyl & $\mathrm{CH}_{3}$ & $\mathrm{H}$ & $\mathrm{C}\left(\mathrm{CH}_{3}\right)_{3}$ & $\mathrm{H}$ & 67 & $53(S)$ \\
\hline
\end{tabular}




\begin{tabular}{cccccccc}
$\mathbf{4 2}$ & $\mathrm{C}\left(\mathrm{CH}_{3}\right)_{3}$ & $\mathrm{Br}$ & $\mathrm{H}$ & $\mathrm{C}\left(\mathrm{CH}_{3}\right)_{3}$ & $\mathrm{H}$ & 98 & $58(S)$ \\
\hline \multicolumn{1}{l}{ Table 1. Continued } & & & & & & \\
\hline $\mathbf{4 3}$ & $\mathrm{Br}$ & $\mathrm{Br}$ & $\mathrm{H}$ & $\mathrm{C}\left(\mathrm{CH}_{3}\right)_{3}$ & $\mathrm{H}$ & 44 & $57(S)$ \\
$\mathbf{4 4}$ & $\mathrm{C}\left(\mathrm{CH}_{3}\right)_{3}$ & $\mathrm{C}\left(\mathrm{CH}_{3}\right)_{3}$ & $\mathrm{H}$ & $\mathrm{C}\left(\mathrm{CH}_{3}\right)_{3}$ & $\mathrm{H}$ & 73 & $59(S)$ \\
$\mathbf{4 5}$ & $\mathrm{C}\left(\mathrm{CH}_{3}\right)_{3}$ & $\mathrm{C}\left(\mathrm{CH}_{3}\right)_{3}$ & $\mathrm{H}$ & $\mathrm{CH}_{2} \mathrm{CH}_{3}$ & $\mathrm{H}$ & 65 & $41(S)$ \\
$\mathbf{4 6}$ & $\mathrm{Br}$ & $\mathrm{Br}$ & $\mathrm{H}$ & $\mathrm{CH}_{2} \mathrm{CH}_{3}$ & $\mathrm{H}$ & 82 & $49(R)$ \\
$\mathbf{4 7}$ & $\mathrm{H}$ & $\mathrm{Br}$ & $\mathrm{H}$ & $\mathrm{CH}_{2} \mathrm{CH}_{3}$ & $\mathrm{H}$ & 66 & $44(R)$ \\
$\mathbf{4 8}$ & $\mathrm{H}$ & $\mathrm{Br}$ & $\mathrm{H}$ & $\mathrm{CH}_{3}$ & $\mathrm{H}$ & 83 & $31(S)$ \\
$\mathbf{4 9}$ & $\mathrm{Br}$ & $\mathrm{Br}$ & $\mathrm{H}$ & $\mathrm{CH}_{3}$ & $\mathrm{H}$ & 78 & $48(S)$ \\
$\mathbf{5 0}$ & $\mathrm{Br}$ & $\mathrm{Br}$ & $\mathrm{H}$ & $\mathrm{H}$ & $\mathrm{CH}$ & 82 & $42(S)$ \\
\hline
\end{tabular}

isolated the active intermediate in the Schiff base-vanadium catalyzed oxidation of sulfide to sulfoxide. The intermediate was found to be a 2:1 complex of Schiff base ligand to vanadium, which then reacts with hydrogen peroxide, eliminating one of the ligands to give a vanadium hydroperoxide complex, which then oxidizes the sulfide to sulfoxide. It is reasonable to assume that a certain amount of steric crowding around the metal in the transition state is essential in order to enhance the enantioselectivity of the sulfide oxidation. From our and previous works related to trimethylsilylcyanation of aldehyde and sulfide to sulfoxide oxidation using Schiff base ligands, it appears that a tert-butyl substituent provides the ideal steric size and gives good enantioselectivity in both types of reactions.

Having investigated the size of substituents on the Schiff base ligands and their effects on the enantioselectivity, we next turned our attention to studying the electronic effects of these substituents in the sulfide to sulfoxide oxidation. Skarzewski and coworkers have reported that with the electron withdrawing nitro group para to the phenolic $\mathrm{OH}$ in the Schiff base-V(IV) complex(system) gives high enantioselectivity in the sulfide to sulfoxide oxidation. ${ }^{32}$ However, in our hands $\mathrm{R}_{2}=\mathrm{NO}_{2}$ and $\mathrm{R}_{1}=$ tert-butyl led to low ee, which is also in agreement with Ellmann's observation. ${ }^{14}$ When the strong electron withdrawing nitro was replaced with a less electron attractive bromine atom, along with sterically bulky substituents $R_{1}, R_{4}$ and $R_{5}$ on the Schiff base ligand, enantioselectivity was improved (ligand 18). A similar trend was also seen in the trimethylsilylcyanation of benzalaldehyde catalyzed by Schiff base-Ti(IV) complex. ${ }^{39}$

In conclusion, the steric requirements of the Schiff base-vanadium (IV) complex-catalyzed oxidation of a sulfide to a chiral sulfoxide parallels the Ti(IV)-Schiff base catalyzed trimethylsilyl cyanation reactions. ${ }^{38}$ Added to this, the presence of electron- withdrawing bromine at $\mathrm{R}_{1}$ or $\mathrm{R}_{2}$ along with appropriate bulky substituents on the ligand enhances the enantioselectivity in the sulfide to sulfoxide oxidation. Thus, in designing new chiral Schiff base ligand-vanadium complexes for the sulfide to sulfoxide oxidation, consideration has to be given to both steric and electronic factors. 


\section{Experimental Section}

\section{General procedure for the oxidation of methyl phenyl thio ether to sulfoxide}

The enantiomeric excesses were determined using a Hewlett-Packard liquid chromatograph (detecting UV diodes at $254 \mathrm{~nm}$ ), with a $(R, R)$-WHELK-01 chiral column.

In a $25 \mathrm{~mL}$ flask were placed vanadyl acetylacetonate $(5.2 \mathrm{mg}, 0.02 \mathrm{mmol})$, the ligand $\mathbf{1 0}$ $(1 R, 2 S)-(+)-1-\left[\mathrm{N}-\left(3^{\prime}\right.\right.$-(1'”-adamantyl)-5'-methylsalicylidene)amino]-2-indanol, $\quad$ (0.012g, 0.03 mmol) along with $4 \mathrm{~mL} \mathrm{CH}_{2} \mathrm{Cl}_{2}$, and the solution was stirred for $5 \mathrm{~min}$ at room temperature. To the stirring solution methylphenylsulfide was added $(0.240 \mathrm{~g}, 2 \mathrm{mmol})$ and the mixture was then cooled to $0{ }^{\circ} \mathrm{C}$, adding $30 \% \mathrm{H}_{2} \mathrm{O}_{2}(0.124 \mathrm{~g}, 1.1 \mathrm{mmol})$ slowly. The mixture was stirred for $20 \mathrm{~h}$ at $0{ }^{\circ} \mathrm{C}$, after which a second portion of [VO(acac) $)_{2}$ (5.2 mg, $\left.0.02 \mathrm{mmol}\right)$ was added, more ligand 10 (12 $\mathrm{mg}, 0.03 \mathrm{mmol}$ ) and $30 \% \mathrm{H}_{2} \mathrm{O}_{2}(124 \mathrm{mg}, 1.1 \mathrm{mmol})$, stirring for an additional $20 \mathrm{~h}$ period at 0 ${ }^{\circ} \mathrm{C}$. The mixture was then extracted with $\mathrm{CH}_{2} \mathrm{Cl}_{2}(2 \times 5 \mathrm{~mL})$, the organic extracts were combined and washed with $\mathrm{H}_{2} \mathrm{O}$, brine and dried over $\mathrm{Na}_{2} \mathrm{SO}_{4}$. The solvent was removed under reduced pressure to give a dark brown liquid. HPLC retention times for the methylphenyl sulfoxides (R) $=26.9 \mathrm{~min}$ and $(\boldsymbol{S})=29.2 \mathrm{~min}$ (hexane:2-propanol, 95:5).

Details of the general procedure for the synthesis and characterization of the Schiff base ligands 10, 11, 15-17, 19-23, 29-32, 37-41 and 44 were reported in previous work. ${ }^{37,38}$

(1R,2S)-(+)-1-[N-(5'-Nitrosalicylidene)amino]-2-indanol (12). Orange solid (0.77 g, 86\%); mp 216-218 ${ }^{\circ} \mathrm{C}$; $[\alpha]^{25}{ }_{\mathrm{D}}=+60.76^{\circ}\left(\mathrm{c}=0.5, \mathrm{CH}_{2} \mathrm{Cl}_{2}\right)$. IR $(\mathrm{KBr}) 3214,2939,2898,1655,1610,1545$, 1329 and $749 \mathrm{~cm}^{-1}$; ${ }^{1} \mathrm{H}$ NMR (200 MHz, $\left.\mathrm{CDCl}_{3}\right): \delta$ 8.68(s, $\left.1 \mathrm{H}\right), 8.40(\mathrm{~d}, 1 \mathrm{H}, \mathrm{J}=2.9 \mathrm{~Hz}), 8.08(\mathrm{dd}$, $1 \mathrm{H}, \mathrm{J}_{1}=2.9 \mathrm{~Hz}$ and $\left.\mathrm{J}_{2}=9.6 \mathrm{~Hz}\right), 7.34-7.25(\mathrm{~m}, 4 \mathrm{H}), 6.68(\mathrm{~d}, 1 \mathrm{H}, \mathrm{J}=9.6 \mathrm{~Hz}), 5.08(\mathrm{~d}, 1 \mathrm{H}, \mathrm{J}=5.3$ $\mathrm{Hz}$ ), $4.70(\mathrm{q}, 1 \mathrm{H}, \mathrm{J}=4.5 \mathrm{~Hz}), 3.20\left(\mathrm{dd}, 2 \mathrm{H}, \mathrm{J}_{1}=5.3 \mathrm{~Hz}\right.$ and $\left.\mathrm{J}_{2}=16.0 \mathrm{~Hz}\right)$ and $3.12\left(\mathrm{dd}, 2 \mathrm{H}, \mathrm{J}_{1}=5.3\right.$ $\mathrm{Hz}$ and $\mathrm{J}_{2}=16.0 \mathrm{~Hz}$ ) ppm; ${ }^{13} \mathrm{C} \mathrm{NMR}\left(50 \mathrm{MHz}, \mathrm{CDCl}_{3}\right): \delta 174.71,164.79,140.47,137.93$, 135.06, 130.25, 128.17, 128.09, 126.23, 124.69, 123.96, 120.81, 113.87, 72.46, 69.24 and 39.03 ppm. EIMS (m/e): $298\left[\mathrm{M}^{+}\right]$(48), 281(30), 149(40), 132(76), 104(100) and 77(65). Anal. calcd. for $\mathrm{C}_{16} \mathrm{H}_{14} \mathrm{~N}_{2} \mathrm{O}_{4}$ : C, 64.43; $\mathrm{H}$, 4.70. Found: C, 64.50; H, 4.75.

(1R,2S)-(+)-1-[N-(3',5'-Di-methylsalicylidene)amino]-2-indanol (13). Yellow solid (0.86 g, 91.3\%); mp 101-104 ${ }^{\circ} \mathrm{C}$; $[\alpha]^{25}=+42.66^{\circ}\left(\mathrm{c}=0.50, \mathrm{CH}_{2} \mathrm{Cl}_{2}\right)$. IR (KBr) 3320, 2955, 1638 and 743 $\mathrm{cm}^{-1} .{ }^{1} \mathrm{H}$ NMR $\left(200 \mathrm{MHz}, \mathrm{CDCl}_{3}\right): \delta 8.49(\mathrm{~s}, 1 \mathrm{H}), 7.27-7.15(\mathrm{~m}, 4 \mathrm{H}), 7.01(\mathrm{~s}, 1 \mathrm{H}), 6.95(\mathrm{~s}, 1 \mathrm{H})$, 4.73(d, $1 \mathrm{H}, \mathrm{J}=5.2 \mathrm{~Hz}), 4.64(\mathrm{q}, 1 \mathrm{H}, \mathrm{J}=5.4 \mathrm{~Hz}), 3.22\left(\mathrm{dd}, 1 \mathrm{H}, \mathrm{J}_{1}=5.8 \mathrm{~Hz}\right.$ and $\left.\mathrm{J}_{2}=15.8 \mathrm{~Hz}\right)$, 3.07(dd, $1 \mathrm{H}, \mathrm{J}_{1}=5.4 \mathrm{~Hz}$ and $\left.\mathrm{J}_{2}=15.8 \mathrm{~Hz}\right), 2.26(\mathrm{~s}, 3 \mathrm{H})$ and $2.20(\mathrm{~s}, 3 \mathrm{H}) \mathrm{ppm}$; ${ }^{13} \mathrm{C} \mathrm{NMR}(50 \mathrm{MHz}$, $\left.\mathrm{CDCl}_{3}\right): \delta 167.08,157.18,141.00,140.80,135.03,129.55,128.68,127.49,127.14,125.93$, 125.55, 124.95, 117.71, 75.70, 75.42, 39.77, 20.57 and $15.64 \mathrm{ppm}$. EIMS (m/e): 281[M $\left.{ }^{+}\right]$ (82.90), 263(18.17), 149(100), 133(32.01), 91(29.61) and 77(44.45). Anal. calcd. for $\mathrm{C}_{18} \mathrm{H}_{19} \mathrm{NO}_{2}$ : C, 76.86; H, 6.76. Found: C, 76.90; H, 6.77.

(1S,2R)-(-)-1-[N-(3'-tert-Butyl-5'-nitrosalicilidene)amino]-2-indanol (14). Yellow solid (0.48 g, 40.0\%); mp 72-78 ${ }^{\circ} \mathrm{C}$; $[\alpha]^{25}=-29.33^{\circ}\left(\mathrm{c}=0.50, \mathrm{CH}_{2} \mathrm{Cl}_{2}\right)$. IR (KBr) 3419, 3108, 2952, 1638, 1600, 1557, 1316 y $748 \mathrm{~cm}^{-1}$; ${ }^{1} \mathrm{H}$ NMR (200 MHz, $\left.\mathrm{CDCl}_{3}\right) \delta 8.53(\mathrm{~s}, 1 \mathrm{H}), 8.22(\mathrm{~d}, 1 \mathrm{H}, \mathrm{J}=2.8 \mathrm{~Hz})$, 8.16(d, 1H, J= 3.0 Hz), 7.36-7.20(m, 4H), 4.95(d, 1H, J $=5.2 \mathrm{~Hz}), 4.76(\mathrm{q}, 1 \mathrm{H}, \mathrm{J}=5.4 \mathrm{~Hz})$, $3.30\left(\mathrm{dd}, 1 \mathrm{H}, \mathrm{J}_{1}=6.0 \mathrm{~Hz}, \mathrm{~J}_{2}=16.0 \mathrm{~Hz}\right), 3.11\left(\mathrm{dd}, 1 \mathrm{H}, \mathrm{J}_{1}=5.0 \mathrm{~Hz}, \mathrm{~J}_{2}=16.0 \mathrm{~Hz}\right.$ ) y $1.42(\mathrm{~s}, 9 \mathrm{H}) \mathrm{ppm}$; 
${ }^{13} \mathrm{C}$ NMR (50 MHz, $\left.\mathrm{CDCl}_{3}\right) \delta 165.04,169.10,140.37,140.44,139.24,138.30,129.24,127.48$, 127.21, 125.81, 125.54, 125.03, 116.70, 74.92, 73.45, 39.47, 35.21 and 28.89 ppm. EIMS (m/e): 354[M+'] (82), 337(18), 221(43), 207(37), 179(42), 133(100), 105(53), 91(31) у 77(39).

(1R,2S)-(+)-1-[N-(3'-tert-Butyl-5'-bromosalicylidene)amino]-2-indanol (18). Yellow oil (1.13 g, 87.4\%); $[\alpha]^{25}{ }_{\mathrm{D}}=+11.03^{\circ}$ (c=0.5, $\mathrm{CH}_{2} \mathrm{Cl}_{2}$ ). IR (neat) 3374, 2951, 1625, 749 and $600 \mathrm{~cm}^{-1} .{ }^{1} \mathrm{H}$ NMR (200 MHz, $\left.\mathrm{CDCl}_{3}\right): \delta 8.47(\mathrm{~s}, 1 \mathrm{H}), 7.44(\mathrm{~d}, 1 \mathrm{H}, \mathrm{J}=2.6 \mathrm{~Hz}), 7.32(\mathrm{~d}, 1 \mathrm{H}, \mathrm{J}=2.6 \mathrm{~Hz}), 7.34-$ 7.21(m, 4H), 4.73(d, $1 \mathrm{H}, \mathrm{J}=5.2 \mathrm{~Hz}), 4.61(\mathrm{q}, 1 \mathrm{H}, \mathrm{J}=5.2 \mathrm{~Hz}), 3.18\left(\mathrm{dd}, 1 \mathrm{H}, \mathrm{J}_{1}=5.6 \mathrm{~Hz}\right.$ and $\mathrm{J}_{2}=$ $15.8 \mathrm{~Hz}), 3.05\left(\mathrm{dd}, 1 \mathrm{H}, \mathrm{J}_{1}=5.2 \mathrm{~Hz}\right.$ and $\left.\mathrm{J}_{2}=15.8 \mathrm{~Hz}\right)$ and $1.36(\mathrm{~s}, 9 \mathrm{H}) \mathrm{ppm} ;{ }^{13} \mathrm{C} \mathrm{NMR}(50 \mathrm{MHz}$, $\left.\mathrm{CDCl}_{3}\right): \delta 165.56,159.57,140.42,140.05,139.98,132.47,131.69,128.34,126.75,125.18$, 124.57, 119.53, 109.54, 74.88, 74.78, 50.28, 39.26, 34.94, 29.22 and 29.00 ppm. EIMS (m/e): 387[ $\left.\mathrm{M}^{+}\right]$(69), 240(24), 133(100), 103(77) and 77(77).

(R)-(+)-2-[N-(5'-Bromosalicylidene)amino]-2-phenyl-1-ethanol (24). Yellow solid (0.35 g, 98 \%); mp 118-120 ${ }^{\circ} \mathrm{C}$; $[\alpha]^{25}{ }_{\mathrm{D}}=+34.0^{\circ}$ (c=0.012, $\mathrm{CH}_{2} \mathrm{Cl}_{2}$ ). IR (KBr) 3406, 2909, 2861, 1623, 768 and $700 \mathrm{~cm}^{-1}$; ${ }^{1} \mathrm{H}$ NMR (200 MHz, $\left.\mathrm{CDCl}_{3}\right) \delta 8.37(\mathrm{~s}, 1 \mathrm{H}), 7.41-7.25(\mathrm{~m}, 7 \mathrm{H}), 6.86(\mathrm{~d}, 1 \mathrm{H}, \mathrm{J}=8.0$ $\mathrm{Hz}), 4.47(\mathrm{t}, 1 \mathrm{H}, \mathrm{J}=6.4 \mathrm{~Hz})$ and $3.93(\mathrm{~d}, 2 \mathrm{H}, \mathrm{J}=6.2 \mathrm{~Hz}) \mathrm{ppm} ;{ }^{13} \mathrm{C} \mathrm{NMR}\left(50 \mathrm{MHz}, \mathrm{CDCl}_{3}\right) \delta$ 164.97, 160.05, 138.80, 135.32, 133.82, 128.94, 128.09, 127.09, 120.04, 119.04, 110.35, 75.74 and 67.58 ppm. Anal. calcd. for $\mathrm{C}_{15} \mathrm{H}_{14} \mathrm{NO}_{2} \mathrm{Br}$ : C, 56.25; H, 4.37. Found: C, 56.30; H, 4.41.

(R)-(+)-2-[N-(3',5'-Dibromosalicylidene)amino]-2-phenyl-1-ethanol (25). Brown oil (0.23 g, 81\%); $[\alpha]^{25}{ }_{\mathrm{D}}=+106.0^{\circ}$ (c=0.032, $\mathrm{CH}_{2} \mathrm{Cl}_{2}$ ). IR (neat) 3345, 2928, 2872, 1636, 736 and $700 \mathrm{~cm}^{-1}$; ${ }^{1} \mathrm{H}$ NMR $\left(200 \mathrm{MHz}, \mathrm{CDCl}_{3}\right) \delta 8.30(\mathrm{~s}, 1 \mathrm{H}), 7.65(\mathrm{~d}, 1 \mathrm{H}, \mathrm{J}=2.4 \mathrm{~Hz}), 7.30(\mathrm{~d}, 1 \mathrm{H}, \mathrm{J}=2.4 \mathrm{~Hz}), 7.42-$ 7.33(m, 5H), 4.55(t, 1H, J= 7.4 Hz), 3.96(d, 1H, J=5.2 Hz), 3.93(d, 1H, J= $2.0 \mathrm{~Hz}) \mathrm{ppm} ;{ }^{13} \mathrm{C}$ NMR $\left(50 \mathrm{MHz}, \mathrm{CDCl}_{3}\right) \delta$ 164.06, 160.50, 138.16, 137.11, 133.14, 128.94, 128.24, 126.75, 118.33, 113.47, 108.14, 73.34 and 67.0 ppm. Anal. calcd. for $\mathrm{C}_{15} \mathrm{H}_{131} \mathrm{NO}_{2} \mathrm{Br}_{2}$ : C, 45.11; H, 3.26. Found: C, 45.14; H, 3.30.

(R)-(+)-2-[ $\boldsymbol{N}$-(3'-tert-Butyl-5-bromosalicylidene)amino]-2-phenyl-1-ethanol (26). Yellow oil (0.15 g, $79 \%$ ); $[\alpha]^{25}{ }_{\mathrm{D}}=+78.00^{\circ}$ (c=0.0238, $\mathrm{CH}_{2} \mathrm{Cl}_{2}$ ). IR (neat) 3365, 2952, 2870, 1628 and 750 $\mathrm{cm}^{-1}$; ${ }^{1} \mathrm{H}$ NMR (200 MHz, $\left.\mathrm{CDCl}_{3}\right) \delta$ 8.32(s, 1H), $7.38(\mathrm{~d}, 1 \mathrm{H}, \mathrm{J}=2.4 \mathrm{~Hz}), 7.35-7.26(\mathrm{~m}, 5 \mathrm{H})$, 7.20(d, 1H, J= 2.4 Hz) 4.39(t, 1H, J=6.0 Hz), 3.84(d, 2H, J=6.0 Hz), 1.40(s, 9H) ppm; ${ }^{13} \mathrm{C} \mathrm{NMR}$ $\left(50 \mathrm{MHz}, \mathrm{CDCl}_{3}\right) \delta 165.97,159.68,140.35,139.19,132.90,132.16,129.10,128.20,127.38$, 120.10, 110.20, 75.76, 67.55, 35.17 and 29.26 ppm. Anal. calcd. for $\mathrm{C}_{19} \mathrm{H}_{22} \mathrm{NO}_{2} \mathrm{Br}$ : C, 60.63; $\mathrm{H}$, 5.85. Found: C, 60.67; H, 5.88.

(R)-(+)-2-[N-(2'-Hydroxy-1-( $N$-naphthalidene)amino]-2-phenyl-1-ethanol (27). Yellow solid (0.052 g, $98 \%$; mp $179-181{ }^{\circ} \mathrm{C} ;[\alpha]^{25}{ }_{\mathrm{D}}=+191.38^{\circ}$ (c=0.02, $\mathrm{CH}_{2} \mathrm{Cl}_{2}$ ). IR (KBr) 3222, 2930, 1630, 1060 and $754 \mathrm{~cm}^{-1}$; ${ }^{1} \mathrm{H}$ NMR (200 MHz, $\left.\mathrm{CDCl}_{3}\right) \delta 8.89(\mathrm{~s}, 1 \mathrm{H}), 7.77(\mathrm{~d}, 1 \mathrm{H}, \mathrm{J}=9.0 \mathrm{~Hz})$, 7.47(d, 1H, J=9.0 Hz), 7.43-7.32(m, 7H), 7.16(t, 1H, J= 7.0 Hz), 6.83(d, 1H, J=9.0 Hz), 4.67(dd, $1 \mathrm{H}, \mathrm{J}_{1}=3.5 \mathrm{~Hz}$ and $\left.\mathrm{J}_{2}=7.9 \mathrm{~Hz}\right), 4.04-3.96(\mathrm{~m}, 2 \mathrm{H}) \mathrm{ppm} ;{ }^{13} \mathrm{C}$ NMR $\left(50 \mathrm{MHz}, \mathrm{CDCl}_{3}\right) \delta$ 173.87, 159.48, 138.06, 137.21, 133.61, 129.38, 129.30, 128.48, 128.08, 127.04, 126.67, 123.43, 123.05, 118.39, 107.39, 71.41 and 67.53 ppm. Anal. calcd. for $\mathrm{C}_{19} \mathrm{H}_{17} \mathrm{NO}_{2}$ : C,78.35; H, 5.84. Found: C, 78.40; H, 5.88.

(1S,2R)-(-)-2-[N-(3'-tert-Butyl-5-bromosalicylidene)amino]-1,2-diphenylethanol (28). Yellow solid (0.25 g, 93 \%); mp 56-58 ${ }^{\circ} \mathrm{C} ;[\alpha]^{25}=-33.0^{\circ}\left(\mathrm{c}=0.105, \mathrm{CH}_{2} \mathrm{Cl}_{2}\right.$ ). IR (KBr) 3424, 2952, 2878, 1631, 1045 
and $705 \mathrm{~cm}^{-1}$; ${ }^{1} \mathrm{H}$ NMR (200 MHz, $\mathrm{CDCl}_{3}$ ) $\delta 8.0(\mathrm{~s}, 1 \mathrm{H}), 7.35-7.29(\mathrm{~m}, 11 \mathrm{H}), 7.00(\mathrm{~d}, 1 \mathrm{H}, \mathrm{J}=3.0$ $\mathrm{Hz}$ ), 5.00(d, $1 \mathrm{H}, \mathrm{J}=7.0 \mathrm{~Hz}), 4.47(\mathrm{~d}, 1 \mathrm{H}, \mathrm{J}=7.0 \mathrm{~Hz}), 1.41(\mathrm{~s}, 9 \mathrm{H}) \mathrm{ppm} ;{ }^{13} \mathrm{C} \mathrm{NMR}\left(50 \mathrm{MHz}, \mathrm{CDCl}_{3}\right)$ $\delta$ 165.59, 159.66, 140.29, 132.78, 132.06, 129.04, 128.38, 127.42, 120.08, 109.97, 80.27, 78.48, 35.23 and 29.26 ppm. Anal. calcd. for $\mathrm{C}_{25} \mathrm{H}_{26} \mathrm{Br} \mathrm{NO}_{2}$ : C, 66.37; H, 5.75. Found: C, 66.40; H, 5.77. (S)-(-)-2-[3'-Adamantyl-5-methylsalicylidene)amino]-4methyl-1-pentanol (33). Yellow solid (0.58 g, $83 \%$ ); mp 78-80 ${ }^{\circ} \mathrm{C}[\alpha]^{25}=-58.04^{\circ}$ (c=0.0026, $\mathrm{CH}_{2} \mathrm{Cl}_{2}$ ). IR (KBr) 3357, 2899, 1628, 1022 and $651 \mathrm{~cm}^{-1} ;{ }^{1} \mathrm{H}$ NMR (200 MHz, $\left.\mathrm{CDCl}_{3}\right) \delta$ 8.32(s, $\left.1 \mathrm{H}\right), 7.08(\mathrm{~d}, 1 \mathrm{H}, \mathrm{J}=2.2 \mathrm{~Hz}), 6.91(\mathrm{~d}$, $1 \mathrm{H}, \mathrm{J}=1.6 \mathrm{~Hz}), 3.64\left(\mathrm{~d}, 1 \mathrm{H}, \mathrm{J}_{1}=7.0 \mathrm{~Hz}\right), 3.40-3.27(\mathrm{~m}, 1 \mathrm{H}), 2.28$ (s, 3H), 2.16(s, 6H), 2.07(s, 3H), 1.79(s, 6H), 1.61-1.52(m, 1H), 1.40-1.22(m, 2H), 0.91(d, 3H, J=6.2 Hz). 0.86(d, 3H, J=6.2 Hz) ppm; ${ }^{13} \mathrm{C}$ NMR (50 MHz, $\left.\mathrm{CDCl}_{3}\right) \delta$ 166.43, 158.38, 137.40, 130.63, 129.62, 126.83, 118.18, 70.02, 66.53, 40.87, 40.30, 37.13, 36.90, 29.05, 24.37, 23.53, 21.50 and 20.67 ppm. Anal. calcd. for $\mathrm{C}_{24} \mathrm{H}_{35} \mathrm{NO}_{2}$ : C, 78.04; H, 9.48. Found: C, 78.05; H, 9.49.

(S)-(-)-2-[N-(3',5'-Di-tert-amylsalicylidene)amino]-4-methyl-1-pentanol (34). Yellow liquid (0.58 g, 93\%); $[\alpha]^{25}{ }_{\mathrm{D}}=-54.5^{\circ}\left(\mathrm{c}=0.020, \mathrm{CH}_{2} \mathrm{Cl}_{2}\right.$ ). IR (neat) 3397, 2956, 2880, 1628, 1061 and $733 \mathrm{~cm}^{-1} ;{ }^{1} \mathrm{H}$ NMR (200 MHz, $\left.\mathrm{CDCl}_{3}\right) \delta$ 8.39(s, 1H), 7.26(d, 1H, J= $\left.2.3 \mathrm{~Hz}\right), 7.05(\mathrm{~d}, 1 \mathrm{H}, \mathrm{J}=2.3 \mathrm{~Hz}), 3.75-3.60(\mathrm{~m}$, $2 \mathrm{H})$, 3.44-3.31(m, 1H), 1.92(q, 1H, J= 7.4 Hz, 1.67-1.49(m, 4H), 1.39(s, 6H), 1.27(s, 6H), 0.93(d, 3H, J=6.4 Hz), 0.89(d, 3H, J=6.6 Hz), 0.69(t, 3H, J= 7.4 Hz), 0.67(t, 3H, J= 7.4 Hz) ppm;

${ }^{13} \mathrm{C}$ NMR (50 MHz, $\mathrm{CDCl}_{3}$ ) $\delta 167.17,158.35,138.59,135.29,129.39,127.20,117.86,70.21$, 66.88, 41.24, 38.78, 37.45, 37.03, 32.87, 28.65, 27.60, 24.58, 23.66, 21.83, 9.63 and 9.29 ppm. Anal. calcd. for $\mathrm{C}_{23} \mathrm{H}_{39} \mathrm{NO}_{2}$ : C, 76.45; $\mathrm{H}, 10.80$. Found: C, 76.48; H, 10.84 .

(S)-(-)-2-\{ $N$-[3',5'-Bis( $\alpha, \alpha$-dimethylbencyl)salicylidene]amino\}-4-methyl-1-pentanol (35). Yellow liquid (0.84 g, 98\%); $[\alpha]^{25}{ }_{\mathrm{D}}=-37.98^{\circ}$ (c=0.0021, $\mathrm{CH}_{2} \mathrm{Cl}_{2}$ ). IR (neat) 3397, 2956, 1623, 1028 and 695 $\mathrm{cm}^{-1}$; ${ }^{1} \mathrm{H}$ NMR $\left(200 \mathrm{MHz}, \mathrm{CDCl}_{3}\right) \delta 8.23(\mathrm{~s}, 1 \mathrm{H}), 7.36-7.06(\mathrm{~m}, 12 \mathrm{H}), 3.60-3.28(\mathrm{~m}, 2 \mathrm{H}), 3.38-$ 3.10(m, 1H), 1.71(s, 9H), 1.61(s, 3H), 1.60-1.10(m, 3H), 0.85(d, 3H, J= 5.6 Hz), 0.82(d, 3H, J= $5.2 \mathrm{~Hz}) \mathrm{ppm} ;{ }^{13} \mathrm{C}$ NMR (50 MHz, $\left.\mathrm{CDCl}_{3}\right) \delta 166.50,165.93,158.41,150.98,139.95,136.54$, 129.74, 129.25, 128.67, 128.51, 128.09, 128.02, 127.30, 127.03, 126.76, 126.26, 126.16, 125.62, 118.16, 42.75, 42.41, 41.07, 31.51, 31.02, 30.57, 28.89, 24.79, 23.86, 23.66, 22.06 and 21.88 ppm. Anal. calcd. for $\mathrm{C}_{31} \mathrm{H}_{31} \mathrm{NO}_{2}$ : C, 81.40; H, 8.53. Found: C, 81.44; H, 8.55.

(S)-(-)-2-[N-(3',5'-Di-tert-butylsalicylidene)amino]-3-methyl-1-pentanol (36). Yellow liquid (0.295 g, $85 \%$ ); $[\alpha]^{25}{ }_{\mathrm{D}}=-39.26$ (c=0.027g, $\mathrm{CH}_{2} \mathrm{Cl}_{2}$ ); IR(neat) 3372, 2959, 28751631 and $704 \mathrm{~cm}^{-1} ;{ }^{1} \mathrm{H}$ NMR (200 MHz, $\left.\mathrm{CDCl}_{3}\right) \delta$ 8.37(s, 1H), 7.39(d, 1H, J=2.3 Hz), 7.12(d, 1H, J= $2.3 \mathrm{~Hz}$ ), 3.803.73 (m, 2H), 3.18-3.05(m, 1H), 1.80-1.54(m, 1H), 1.45(s, 9H), 1.31(s, 9H), 1.26-1.12(m, 2H), 0.96-0.82(m, 6H) ppm; ${ }^{13} \mathrm{C}$ NMR $\left(50 \mathrm{MHz} \mathrm{CDCl}_{3}\right) \delta 167.17,158.44,140.43,136.99,127.35$, 126.40, 118.00, 77.23, 64.54, 37.17, 35.30, 34.40, 31.77, 29.72, 25.70, 16.13 and 11.63 ppm.

(S)-(-)-2-[N-(3'-tert-Butyl-5-bromosalicylidene)amino]-3,3-dimethyl-1-butanol (42). Yellow solid (0.37 g, $89 \%$ ); mp 141-143 ${ }^{\circ} \mathrm{C}$; $[\alpha]^{25}{ }_{\mathrm{D}}=-37.0^{\circ}$ (c=0.030, $\mathrm{CH}_{2} \mathrm{Cl}_{2}$ ); IR (KBr) 3441, 2962 and $1626 \mathrm{~cm}^{-1} ;{ }^{1} \mathrm{H}$ NMR (500 MHz, $\left.\mathrm{CDCl}_{3}\right) \delta$ 13.95(s, 1H), 8.22(s, 1H), $7.30(\mathrm{~d}, 1 \mathrm{H}, \mathrm{J}=2.4 \mathrm{~Hz}$ ), 7.22(d, $1 \mathrm{H}, \mathrm{J}=2.4 \mathrm{~Hz}), 3.92\left(\mathrm{dd}, 1 \mathrm{H}, \mathrm{J}_{1}=2.56 \mathrm{~Hz}\right.$ and $\left.\mathrm{J}_{2}=11.1 \mathrm{~Hz}\right) 3.72(\mathrm{t}, 1 \mathrm{H}, \mathrm{J}=9.65 \mathrm{~Hz})$, 2.93(dd, $1 \mathrm{H}, \mathrm{J}=2.8 \mathrm{~Hz}, \mathrm{~J}=9.52 \mathrm{~Hz}), 1.40(\mathrm{~s}, 9 \mathrm{H}), 1.00(\mathrm{~s}, 9 \mathrm{H}) \mathrm{ppm} ;{ }^{13} \mathrm{C} \mathrm{NMR}\left(50 \mathrm{MHz}, \mathrm{CDCl}_{3}\right) \delta$ 165.60, 159.88, 140.35, 132.58, 131.99, 120.01, 110.01, 81.49, 62.62, 35.32, 33.44, 29.40 and 27.27 ppm. Anal. calcd. for $\mathrm{C}_{17} \mathrm{H}_{26} \mathrm{Br} \mathrm{NO}_{2}$ : C, 57.30; H, 7.30. Found: C, 57.33; H, 7.34. 
(S)-(-)-2-[N-(3',5'-Dibromosalicylidene)amino]-3,3-dimethyl-1-butanol (43). Yellow solid (0.28 g, $98 \%$ \%) mp 138-140 ${ }^{\circ} \mathrm{C}[\alpha]^{25}{ }_{\mathrm{D}}=-34.28$ (c=0.00245g, $\mathrm{CH}_{2} \mathrm{Cl}_{2}$ ); IR (KBr) 3365, 2959, 2878, 1638, 1085, 857 y $753 \mathrm{~cm}^{-1} ;{ }^{1} \mathrm{H}$ NMR $\left(200 \mathrm{MHz}, \mathrm{CDCl}_{3}\right) \delta 8.083(\mathrm{~s}, 1 \mathrm{H}), 7.54$ (d, $1 \mathrm{H}, \mathrm{J}=2.4 \mathrm{~Hz}$ ), 7.20(d, $1 \mathrm{H}, \mathrm{J}=2.4 \mathrm{~Hz}$ ), 4.039-3.96(dd, $1 \mathrm{H}, \mathrm{J}_{1}=3.0 \mathrm{~Hz}, \mathrm{~J}_{2}=11.2 \mathrm{~Hz}$ ), 3.73-3.62(dd, $1 \mathrm{H}, \mathrm{J}_{1}=9.6$ $\mathrm{Hz}, \mathrm{J}_{2}=11.5 \mathrm{~Hz}$ ), 3.13-3.08(dd, $1 \mathrm{H}, \mathrm{J}_{1}=2.4 \mathrm{~Hz}, \mathrm{~J}_{2}=9.2 \mathrm{~Hz}$ ), $1.00(\mathrm{~s}, 9 \mathrm{H}) \mathrm{ppm} ;{ }^{13} \mathrm{C}$ NMR (50 $\left.\mathrm{MHz}, \mathrm{CDCl}_{3}\right) \delta 164.87,164.65,139.14,133.70,116.86,115.51,106.37,77.98,61.64,32.84$ and 26.84 ppm. Anal. calcd. for $\mathrm{C}_{13} \mathrm{H}_{17} \mathrm{NO}_{2} \mathrm{Br}_{2}$ : C, 41.16; H, 4.48, Found: C, 41.46; H, 4.78.

(S)-(-)-2-[N-(3',5'-Di-tert-butylsalicylidene)amino]-1-butanol (45). Yellow solid (1.40 g, $92 \%$ ); mp 78-80 ${ }^{0} \mathrm{C}[\alpha]^{25}{ }_{\mathrm{D}}=-22.46^{\circ}\left(\mathrm{c}=0.00276, \mathrm{CH}_{2} \mathrm{Cl}_{2}\right.$ ). IR (KBr) 3285, 2955, 2872, 1632, 1062, 831 and $687 \mathrm{~cm}^{-1}$; ${ }^{1} \mathrm{H}$ NMR $\left(200 \mathrm{MHz}, \mathrm{CDCl}_{3}\right) \delta 8.40(\mathrm{~s}, 1 \mathrm{H}), 7.40(\mathrm{~d}, 1 \mathrm{H}, \mathrm{J}=2.4 \mathrm{~Hz}$ ), 7.13(d, $1 \mathrm{H}, \mathrm{J}=2.2 \mathrm{~Hz}$ ), 3.77-3.64(m, 1H, Hz), 3.24-3.11(m, 1H), 1.76-1.51(m, 2H), 1.45(s, 9H), 1.31(s, 9H), 0.91(t, 3H, J= 7.4 Hz) ppm; ${ }^{13} \mathrm{C}$ NMR (50 MHz, $\left.\mathrm{CDCl}_{3}\right) \delta 166.87,158.07,140.20$, 136.70, 127.10, 126.10, 117.67, 73.64, 66.16, 35.01, 34.13, 31.84, 29.44, 25.16 and $10.67 \mathrm{ppm}$. Anal. calcd. for $\mathrm{C}_{19} \mathrm{H}_{31} \mathrm{NO}_{2}$ : C, 74.75; H, 10.16. Found: C, 74.79; H, 10.19 .

(R)-(+)-2-[N-(3',5'-Dibromosalicylidene)amino]-1-butanol (46). Yellow solid (0.52 g, 81\%); mp 137-139 ${ }^{\circ} \mathrm{C} ;[\alpha]^{25}{ }_{\mathrm{D}}=+20.00^{\circ}$ (c=0.035, $\mathrm{CH}_{2} \mathrm{Cl}_{2}$ ). IR (KBr) 3264, 2968, 2853, 1648, 1068, 756 and $691 \mathrm{~cm}^{-1}$; ${ }^{1} \mathrm{H}$ NMR $\left(200 \mathrm{MHz}, \mathrm{CDCl}_{3}\right) \delta 8.17(\mathrm{~s}, 1 \mathrm{H}), 7.62(\mathrm{~d}, 1 \mathrm{H}, \mathrm{J}=2.1 \mathrm{~Hz}), 7.26(\mathrm{~d}$, $1 \mathrm{H}, \mathrm{J}=2.1 \mathrm{~Hz}$ ), $3.84\left(\mathrm{dd}, 1 \mathrm{H}, \mathrm{J}_{1}=3.8 \mathrm{~Hz}\right.$ and $\left.\mathrm{J}_{2}=11.4 \mathrm{~Hz}\right), 3.67\left(\mathrm{dd}, 1 \mathrm{H}, \mathrm{J}_{1}=8.4 \mathrm{~Hz}\right.$ and $\mathrm{J}_{2}=$ $11.2 \mathrm{~Hz}$ ), 3.41-3.28(m, 1H), 1.80-1.50(m, 2H), 0.95(t, 3H, J= 7.6 Hz) ppm; ${ }^{13} \mathrm{C}$ NMR (50 MHz, $\left.\mathrm{CDCl}_{3}\right) \delta 167.91,142.36,137.00,81.29,80.66,73.77,68.93,28.13$ and 14.23 ppm. Anal. calcd. for $\mathrm{C}_{11} \mathrm{H}_{13} \mathrm{NO}_{2} \mathrm{Br}_{2}$ : C, 37.60; H, 3.70. Found: C, 37.65; H, 3.74.

(R)-(+)-2-[N-(5'-Bromosalicylidene)amino]-1-butanol (47). Yellow solid (0.49 g, $99 \%)$; mp 57-59 ${ }^{0} \mathrm{C} ;[\alpha]^{25}{ }_{\mathrm{D}}=+22.65^{\circ}\left(\mathrm{c}=0.064, \mathrm{CH}_{2} \mathrm{Cl}_{2}\right)$. IR $(\mathrm{KBr}) 3284,2925,2872,1633,765$ and $627 \mathrm{~cm}^{-1} ;{ }^{1} \mathrm{H}$ NMR $\left(200 \mathrm{MHz}, \mathrm{CDCl}_{3}\right) \delta 8.26(\mathrm{~s}, 1 \mathrm{H}), 7.39-7.32(\mathrm{~m}, 2 \mathrm{H}), 6.81\left(\mathrm{dd}, 1 \mathrm{H}, \mathrm{J}_{1}=2.6 \mathrm{~Hz}\right.$, $\left.\mathrm{J}_{2}=6.8 \mathrm{~Hz}\right), 3.80-3.60(\mathrm{~m}, 2 \mathrm{H}), 3.26-3.12(\mathrm{~m}, 1 \mathrm{H}), 1.72-1.48(\mathrm{~m}, 2 \mathrm{H}), 0.89(\mathrm{t}, 3 \mathrm{H}, \mathrm{J}=7.4 \mathrm{~Hz}) \mathrm{ppm}$; ${ }^{13} \mathrm{C}$ NMR (50 MHz, $\left.\mathrm{CDCl}_{3}\right) \delta 164.58,160.94,135.35,133.83,120.08,119.40,110.19,73.34,65.90$, 25.14 and 10.58 ppm. Anal. calcd. for $\mathrm{C}_{11} \mathrm{H}_{14} \mathrm{NO}_{2} \mathrm{Br}$ : C, 48.52; H, 5.14. Found: C, 48.58; H, 5.18.

(S)-(+)-2-[5'-Bromosalicylidene)amino]-1-propanol (48). Yellow solid (0.32 g, 56\%); mp 49$51{ }^{\circ} \mathrm{C}$; $[\alpha]^{25}{ }_{\mathrm{D}}=+19.00^{\circ}\left(\mathrm{c}=0.051, \mathrm{CH}_{2} \mathrm{Cl}_{2}\right.$ ). IR $(\mathrm{KBr}) 3380,2930,2870,1633$ and $625 \mathrm{~cm}^{-1} ;{ }^{1} \mathrm{H}$ NMR $\left(200 \mathrm{MHz}, \mathrm{CDCl}_{3}\right) \delta 8.29(\mathrm{~s}, 1 \mathrm{H}), 7.40-7.30(\mathrm{~m}, 2 \mathrm{H}), 6.82(\mathrm{~d}, 1 \mathrm{H}, \mathrm{J}=7.8 \mathrm{~Hz}), 3.76-3.40(\mathrm{~m}$, $3 \mathrm{H}), 1.25(\mathrm{~d}, 3 \mathrm{H}, \mathrm{J}=6.4 \mathrm{~Hz}) \mathrm{ppm} ;{ }^{13} \mathrm{C}$ NMR $\left(50 \mathrm{MHz}, \mathrm{CDCl}_{3}\right) \delta 163.30,160.41,134.78,133.23$, 119.50, 118.91, 109.58, 66.71, 66.09 and 18.03 ppm. Anal. calcd. for $\mathrm{C}_{10} \mathrm{H}_{12} \mathrm{NO}_{2} \mathrm{Br}$ : C,46.51; $\mathrm{H}$, 4.65. Found: C, 46.54; H, 4.67.

(R)-(+)-2-[N-(3',5'-Di-bromosalicylidene)amino]-1-propanol (49). Yellow solid (0.25 g, 81\%); mp 138-140 ${ }^{\circ} \mathrm{C} ;[\alpha]^{25}{ }_{\mathrm{D}}=+29.70^{\circ}\left(\mathrm{C}=0.00303, \mathrm{CH}_{2} \mathrm{Cl}_{2}\right.$ ). IR (KBr) 3274, 2963, 2847, 1650, 754 and $692 \mathrm{~cm}^{-1}$; ${ }^{1} \mathrm{H}$ NMR (200 MHz, $\left.\mathrm{CDCl}_{3}\right) \delta 8.19(\mathrm{~s}, 1 \mathrm{H}), 7.66(\mathrm{~d}, 1 \mathrm{H}, \mathrm{J}=2.4 \mathrm{~Hz}), 7.30(\mathrm{~d}, 1 \mathrm{H}, \mathrm{J}=2.4$ $\mathrm{Hz}$ ), 4.16-3.99(tq, $1 \mathrm{H}, \mathrm{J}_{1}=3.8 \mathrm{~Hz}$ and $\mathrm{J}_{2}=6.8 \mathrm{~Hz}$ ), 3.72(dd, $1 \mathrm{H}, \mathrm{J}_{1}=15.1 \mathrm{~Hz}$ and $\mathrm{J}_{2}=3.6 \mathrm{~Hz}$ ), 3.52(dd, $1 \mathrm{H}, \mathrm{J}_{1}=12.4 \mathrm{~Hz}$ and $\left.\mathrm{J}_{2}=7.2 \mathrm{~Hz}\right), 1.28(\mathrm{~d}, 3 \mathrm{H}, \mathrm{J}=6.2 \mathrm{~Hz}) \mathrm{ppm} ;{ }^{13} \mathrm{C}$ NMR $(50 \mathrm{MHz}$, $\left.\mathrm{CDCl}_{3}\right) \delta 165.11,161.92,138.33,133.25,118.66,114.23,107.42,66.20,63.70,39.98,39.56$ and 20.90 ppm. Anal. calcd. for $\mathrm{C}_{10} \mathrm{H}_{11} \mathrm{NO}_{2} \mathrm{Br}_{2}$ : C, 35.60; H, 3.26. Found: C, 35.64; H, 3.30 . 
(R)-(-)-2-[ $N$-(3',5'-Dibromosalicylidene)amino]-2-propanol (50). Yellow solid (0.57 g, 98\%); mp 125-127 ${ }^{\circ} \mathrm{C} ;[\alpha]^{25}{ }_{\mathrm{D}}=-30.88^{\circ}\left(\mathrm{c}=0.0014, \mathrm{CH}_{2} \mathrm{Cl}_{2}\right.$ ). IR $(\mathrm{KBr}) 3350,2980,1658,1143,1046,870$ and $695 \mathrm{~cm}^{-1}$; ${ }^{1} \mathrm{H}$ NMR $\left(200 \mathrm{MHz}, \mathrm{CDCl}_{3}\right) \delta 7.85(\mathrm{~s}, 1 \mathrm{H}), 7.30(\mathrm{~d}, 1 \mathrm{H}, \mathrm{J}=2.2 \mathrm{~Hz}), 7.00(\mathrm{~d}, 1 \mathrm{H}, \mathrm{J}=$ $2.2 \mathrm{~Hz}$ ), 3.70-3.56(m, $1 \mathrm{H}), 3.33\left(\mathrm{dd}, 1 \mathrm{H}, \mathrm{J}_{1}=3.6 \mathrm{~Hz}\right.$ and $\mathrm{J}_{2}=12.2 \mathrm{~Hz}$ ), 3.15(dd, $1 \mathrm{H}, \mathrm{J}_{1}=7.0 \mathrm{~Hz}$ and $\left.\mathrm{J}_{2}=12.4 \mathrm{~Hz}\right), 0.90(\mathrm{~d}, 3 \mathrm{H}, \mathrm{J}=2.0 \mathrm{~Hz}) \mathrm{ppm} ;{ }^{13} \mathrm{C} \mathrm{NMR}\left(50 \mathrm{MHz}, \mathrm{CDCl}_{3}\right) \delta 164.90,162.60,137.92$, 133.17, 118.08, 114.43, 106.15, 65.59, 62.73 and $20.92 \mathrm{ppm}$. Anal. calcd. for $\mathrm{C}_{10} \mathrm{H}_{11} \mathrm{NO}_{2} \mathrm{Br}_{2}$ : C, 35.60; H, 3.26. Found: C, 35.62; H, 3.29.

\section{Acknowledgements}

We gratefully acknowledge support for this project by COSNET grant 498.01-P(2001), CONACyT grant 37827-E(2001) and graduate fellowship by CONACyT for A.G.

\section{References}

1. Seydon-Penne, J. Chiral Auxilaries and Ligands in Asymmetric Synthesis; Wiley: New York, 1995.

2. Kagan, H. B.; Diter, P. Organosulfur Chemistry; Page, P.C.B., Ed.; Academic Press: New York, 1998, Vol 2, p1.

3. Matta, E. G. Phosphorus, Sulfur and Silicon 1996, 117, 231.

4. Baird, C. P.; Rayner, C.M. J. Chem. Soc., Perkin Trans. I. 1998, 1973.

5. Procter, D. J. J. Chem. Soc., Perkin Trans. I 1999, 641.

6. Carreño, M. C. Chem. Rev. 1995, 95, 1717.

7. Satoh, T.; Kuramochi, Y.; Inoue, Y. Tetrahedron Lett. 1999, 40, 8815.

8. Nakamura, S.; Yasuda, H.; Watanabe, Y.; Toru, T. Tetrahedron Lett. 2000, 41, 4157.

9. Nakamura, S.; Uchiyama, Y.; Ishikawa, S.; Fukinbara, R.; Watanabe, Y.; Toru, T. Tetrahedron Lett. 2002, 43, 2381.

10. Chou, S. S.; Liang, P. W. Tetrahedron Lett. 2002, 43, 4865.

11. Carretero, J. C.; Ruano, J. L. G.; Cabrejas, L. M. M. Tetrahedron: Asymmetry 1997, 8, 2215.

12. Aggarwal, V. K.; Grainger, R. S.; Adams, H.; Spargo, P. L. J. Org. Chem. 1998, 63, 348.

13. Aggarwal, V. K.; Gultekin, R. S.; Grainger, R .S; Adams, H.; Spargo, P. L. J. Chem. Soc., Perkin Trans I 1998, 2771.

14. (a) Cogan, D. A.; Liu, G.; Kim, K.; Backes, B. J.; Ellman, J .A. J. Am. Chem. Soc. 1998, 120, 8011. (b) Owens, T. M.; Souers, A. J.; Ellman, J. A. J. Org. Chem. 2003, 68, 3. (c) Blum, S. A.; Bergman, R. G.; Ellman, J. A. J. Org. Chem. 2003, 68, 150.

15. (a) Tang, T. P.; Volkman, S. K.; Ellman, J. A. J. Org. Chem. 2001, 66, 8772. (b) Yuste, F.; Ortiz, B.; Carrasco, A.; Peralta, M.; Quintero, L.; Sanchez-Obregon, R.; Walls, F.; Ruano, J. L. G. Tetrahedron: Asymmetry 2000, 11, 3097. 
16. (a) Nakamura, S.; Watanabe, Y.; Toru, T. J. Chem. Soc., Perkin Trans I 1999, 3403. (b) Colobert, F.; Tito, A.; Khiar, N.; Denni, D.; Medina, M. A.; Martín-Lomas, M.; Ruano, J. L, G.; Solladié. J. Org. Chem. 1998, 63, 8919. (c) Bravo, P.; Crucianelli, M.; Farina, A.; Meille, S. V.; Volonterio, A.; Zanda, M. Eur. J. Org. Chem. 1998, 435.

17. ( a) Morita, S.; Matsubara, J.; Otsubo, K.; Kitano, K.; Ohtani, T.; Kawano, Y.; Uchida, M. Tetrahedron: Asymmetry 1997, 8, 3707. (b) Carlsson, E.; Lindberg, P.; Unge, S.V. Chemistry in Britain 2002, 38, 42.

18. Matsugi, M.; Fukudo, N.; Muguruma, Y.; Yamaguchi, T.; Minamikawa, J.; Otsuka, S. Tetrahedron 2001, 57, 2739.

19. Naito, S.; Nishimura, M. Yakugaku Zasshi 2001, 121, 989.

20. (a) Cotton, H.; Elebring, T.; Larsson, M.; Li, L.; Sörensen, H.; Unge, S. V. Tetrahedron: Asymmetry 2000, 11, 3819. (b) Padmanabhan, S.; Lavin, R. C.; Durranat, G. J. Tetrahedron: Asymmetry 2000, 11, 3455.

21. Khiar, N.; Fernández, I.; Alcudia, F. Tetrahedron Lett. 1993, 34, 123.

22. Tokunoh, R.; Sodeoka, M.; Aoe, K.; Shibasaki, M. Tetrahedron Lett. 1995, 36, 8035.

23. Allen, J. V.; Bower, F. F.; Williams, J. M. Tetrahedron: Asymmetry 1994, 5, 1895.

24. Bolm, C.; Muller, J. Tetrahedron 1994, 50, 4355.

25. Carreño, M. C.; Ruano, J. L. G.; Maestro, M. C.; Cabrejas, L.M.M. Tetrahedron: Asymmetry 1993, 4, 727.

26. Kagan, H.B. In Catalytic Asymmetric Synthesis, 2nd. Edition, Ojima, I. Ed. Wiley-VCH, Inc. 2000.

27.Yamamoto, K.; Ando, H.; Shuetake, T.; Chikamatsu, H. J. Chem. Soc., Chem. Commun. 1989, 754.

28. Komatsu, K.; Nishibayashi, Y.; Sugata, T.; Unemura, S. Tetrahedron Lett. 1986, 33, 5397.

29. Bolm, C.; Bienewald, F. Synlett 1998, 1327.

30. Colonna, S.; Manfredi, A.; Spadoni, M.; Casella, L.; Gullotti, M. J. Chem. Soc., Perkin Trans. I 1987, 71.

31. Bolm, C.; Bienewald, F. Angew. Chem. Int. Ed. 1995, 34, 2640.

32. Skarzewski, J.; Ostrycharz, E.; Siedlecka, R. Tetrahedron: Asymmetry 1999, 10, 3457.

33. Colombo, A.; Marturano, G.; Pasini, A. Gazz. Chim. Ital. 1986, 116, 35.

34. Nakajima, K.; Sasaki, C.; Kojima, M.; Aoyama, T.; Ohba, S.; Saito, Y.; Fujita, J. Chem. Lett. 1987, 2189.

35. Noda, K.; Hosoya, N.; Irie, R.; Yamashita, Y.; Katsuki, T. Tetrahedron 1994, 50, 9609.

36. Kokubo, C.; Katsuki, T. Tetrahedron 1996, 52, 13895.

37. Z.Flores-Lopez, L.; Parra-Hake, M.; Somanathan, R.; Walsh, P. J. Organometallics 2000, 19, 2153.

38. Gama, A.; Z.Flores-Lopez, L.; Aguirre, G.; Parra-Hake, M.; Somanathan, R.; Walsh, P. J. Tetrahedron: Asymmetry 2002, 13, 149.

39. Gama, A.; Z.Flores-Lopez, L.; Aguirre, G.; Parra-Hake, M.; Somanathan, R.; Walsh, P. J. manuscript in preparation. 